\title{
Layer-2 protocol adaptation method to improve fast handoff for mobile IPv6 vertical handoffs
}

\begin{abstract}
Inter-technology roaming is known as one of the interesting challenges toward fourth generation of mobile and wireless communication. While FMIPv6 standardizes the fast handoff solutions in IP layer, the issues of media independency are being investigated through IEEE802.21 project. The integration of these two standards is believed to result in solutions for vertical handoffs between different network technologies. This paper presents an improved link layer mechanism to assist FMIPv6 for seamless vertical handoffs. We introduce a new access router discovery method and propose a vertical handoff algorithm accordingly. Further, we report the implementation details performed through simulations. The simulations evidence performance improvements in terms of latency and packet loss. It is also analytically shown that by enabling access router discovery method and improving link layer event services, an MN can be well prepared for handoff and perform faster movements.
\end{abstract}

Keyword: Vertical handoff; Heterogeneous networks; FMIPv6; MIH; Access router discovery 